\title{
How Do the Consideration of Non-Normal Return Distributions and of Higher Moments Influence the Optimal Asset Allocation in Swiss Pension Funds?*
}

\author{
Philipp Müller and Joël Wagner ${ }^{\dagger}$
}

\begin{abstract}
The low interest rates that prevail on many capital markets impose great challenges for the asset management of financial organizations. They try to achieve target returns for their clients, a solid one-period funding ratio and a low one-period underfunding probability. In this summarizing contribution of Müller and Wagner (2018), we aim to study the impact of capital allocation strategies for pension funds in Switzerland. Thereby, we compare classic Markowitz theory with an extended Taylor series approach for the utility function. It is further analyzed how the assumption of normally distributed returns drives the optimal asset allocation when compared with using the distributions corresponding to the best fit of the historical data. Taking the extended utility function including the first four central moments and the alternative return distributions, we simulate the assets of a pension fund in a oneperiod model with the Monte Carlo method. A comparison of these results with those obtained from the classic minimum variance theory concludes that a considerable change of the portfolio weights takes place. Our research is relevant for theory and practice alike. Financial institutions can strongly profit from comparing different approaches when assessing their investment strategy.
\end{abstract}

Zusammenfassung Das niedrige Zinsniveau, das auf vielen Kapitalmärkten vorherrscht, stellt das Assetmanagement von Finanzeinrichtungen vor große Herausforderungen. Ihr Ziel ist es, Zielrenditen für ihre Kunden, einen sicheren Deckungsgrad auf Einjahressicht sowie eine niedrige einjährige Unterdeckungswahrscheinlichkeit sicherzustellen. In dieser Zusammenfassung der Arbeit von Müller und Wagner (2018) werden die Strategien zur Kapitalallokation von Schweizer Pensionskassen untersucht. Dabei vergleichen wir die klassische Markowitz Portfoliotheorie mit dem Ansatz einer erweiterten Taylorreihe für die Nutzenfunktion. Des Weiteren wird die Annahme von normalverteilten Renditen für die optimale Vermögensallokation mit Verteilungen verglichen, welche die historischen Werte besser abbilden. Mit Hilfe einer erweiterten Nutzenfunktion, welche die ersten vier zentralen Momente beinhaltet sowie den alternativen Renditeverteilungen, simulieren wir das Kapital einer Pensionskasse in einem Einperiodenmodell. Die Resultate zeigen, dass eine erhebliche Veränderung der Portfoliogewichte stattfindet. Unsere Ergebnisse sind sowohl für die Theorie als auch für die Praxis relevant. Finanzinstitutionen können stark von einem Vergleich verschiedener Ansätze in der Beurteilung der Anlagestrategie profitieren.

\footnotetext{
${ }^{*}$ This is a post-peer-review, pre-copyedit version of an article published in Zeitschrift für die gesamte Versicherungswissenschaft. The final authenticated version is available online at: https://doi.org/10.1007/s12297-018-0410-9.

${ }^{\dagger}$ Philipp Müller (philipp.muller.1@unil.ch) and Joël Wagner (joel.wagner@unil.ch) are with the Department of Actuarial Science, Faculty of Business and Economics, University of Lausanne. The second author is also with the Swiss Finance Institute, Lausanne, Switzerland. Both authors acknowledge financial support from the Swiss National Science Foundation (grant no. 100018_159428).
} 


\section{Remark}

This work summarizes the main findings of a working paper written by Müller and Wagner (2018) that was presented by the authors at the annual meeting of "Deutscher Verein für Versicherungswissenschaft e.V." in Munich, March 2018.

\section{Introduction}

In the current low interest rate environment, a proper asset allocation strategy is crucial for financial institutions like life insurers and pension funds. Often being obliged to grant their clients a guaranteed interest rate on their savings, they face the issue of having to find a suitable combination of asset classes to invest in. With respect to Swiss pension funds, this topic is of great importance. As funds face large obligations towards their members, it is necessary for them to have an investment strategy that balances expected return and investment risk. While the pension funds aim to achieve adequate returns in a competitive market environment, the resulting volatility should not be too high. Otherwise, years with very low capital market returns could lead to a strong decrease of the funding ratio and thus put the fund's solvency at risk. It is therefore crucial to choose a combination of assets that meets the needed returns while maintaining a certain safety level. In our work, we seek to examine this subject by looking at utility-driven asset allocations under selected return distribution assumptions. To this respect, we study the impact that higher moments have when included in the decision taker's utility function. We analyze how using different distributions for simulating the assets leads to a better fit of the historic data and thus to different simulation results. In order to compare the resulting allocations, we simulate the assets of a fund and analyze the results after one period. A comprehensive literature review is presented in Müller and Wagner (2018).

To start our study, we consider a pension fund with a given asset-liability situation and which is regulated by prevalent rules of the Swiss pension fund system. Our aim is to find the asset allocation that allows the fund to reach a given expected target return, a given funding ratio or a given low underfunding probability. Having established an optimal allocation strategy, we simulate the assets of the fund. For this, we first make use of a multivariate normal distribution. However, asset returns exhibit characteristics such as skewness and heavy tails, that cannot be fully reflected by using a normal distribution. Therefore, we perform the simulation also by using alternative distributions taking their specific correlation structure into account. This way, the historic data can be fitted in a more flexible way that should improve the results. With the optimal allocation corresponding to the objective return and a distribution that fits the historic data, we simulate the assets and liabilities of the pension fund in a one-period model. While the assets evolve according to the simulated returns, the liabilities are assumed to be credited with the guaranteed interest rate. Based on the results, it is analyzed in what state the fund is at the end of the period. Among others, this involves examining key figures such as the expected funding ratio, selected quantiles of that ratio and the underfunding probability. In this context, we compare what implications the use of an asset-liability approach has compared to a classical analysis. In this, the asset allocation can be set up with regard to a desired funding ratio or underfunding probability rather than a target return.

The remainder of this summary is organized as follows. Section two introduces the framework that we use for modeling the pension fund and the optimization problem for determining the optimal asset allocation. The third section presents the asset classes together with their descriptive statistics and the fitting of return distributions. Section four presents the optimization and simulation results. This comprises both, the results for the minimum variance portfolio with normally distributed returns as well as the extended utility along with the best-fit return distributions. The final section concludes. 


\section{Model Framework}

In the following, we describe the model framework applied within the analysis conducted by Müller and Wagner (2018). We introduce a simple asset allocation framework and solvency indicators for pension funds. This involves describing the processes that take place within the fund as well as in the assets that it invests in. Then, we develop an optimal portfolio theory in which we use different utility functions that incorporate higher moments.

\subsection{Pension Funds and Key Funding Indicators in a One-Period Model}

For the pension fund, we focus on a simplified representation of the accumulation phase of a defined contribution fund in Switzerland. We examine how the assets $A_{0}$ and the liabilities $L_{0}$ evolve in a oneperiod model from time $t=0$ to $t=1$, given an asset allocation and legal minimum increases of the liabilities. Thereby, we disregard fluctuations, annuitization and deaths. At the end of the period, the state of the fund is analyzed by considering the funding ratio and the probability of underfunding.

The assets $A_{0}$ represent the capital that is available to the fund for investing on the capital market at time zero. In our model, we assume that the fund invests in $n$ different asset classes $i=1, \ldots, n$. The shares $\alpha_{i}$, that are invested in the respective classes $i$, are summarized in the vector $\boldsymbol{\alpha}=\left(\alpha_{1}, \ldots, \alpha_{n}\right)^{\prime}$. These shares are in the focus of the present study. In Switzerland, the regulator imposes limits on the asset shares that can be invested in the various asset types (see BVV2, 2017, Art. 55). Therefore, we introduce an upper limit $\alpha_{i}^{\max }$ for each asset class $i$. We assume that the entire assets are invested and suppose that no short sales are made. Consequently, it holds for the asset shares $\alpha_{i}$ that $0 \leq \alpha_{i} \leq \alpha_{i}^{\max }, i=1, \ldots, n$ and $\sum_{i=1}^{n} \alpha_{i}=1$.

The stochastic asset returns in the period are denoted by $\boldsymbol{r}=\left(r_{1}, \ldots, r_{n}\right)^{\prime}$. They are the only source of risk in our study. With the returns $r_{i}$ denoting the different assets and $\alpha_{i}$ the investment shares, the overall portfolio return is

$$
r_{\mathrm{A}}=\boldsymbol{\alpha}^{\prime} \cdot \boldsymbol{r}=\sum_{i=1}^{n} \alpha_{i} \cdot r_{i} .
$$

Starting with a value of $A_{0}$ at time $t=0$ and considering continuous compounding of the interest return, the value of the assets at time $t=1$ is given by

$$
A_{1}=A_{0} \cdot e^{r_{\mathrm{A}}}=A_{0} \cdot e^{\sum_{i=1}^{n} \alpha_{i} \cdot r_{i}} .
$$

The liabilities $L_{t}$ represent the obligations that the fund has towards its members. This includes the regular contributions that have been paid by the actives as well as surpluses that the fund can distribute when being in good health. In the Swiss system, funds are required to credit their members at least a minimum interest rate $r_{\mathrm{L}}$ on the compulsory part of their second pillar pension savings. The value of $r_{\mathrm{L}}$ is set by the legislator at the end of every year according to the prevailing conditions on the financial market (see BVV2, 2017, Art. 12). In our model, we assume that the liabilities start with a value of $L_{0}$ at time zero and are compounded with $r_{\mathrm{L}}$ over the course of the period. Their value at time one therefore is

$$
L_{1}=L_{0} \cdot e^{r_{\mathrm{L}}} .
$$

While in our model $L_{1}$ is deterministic (no fluctuations, no mortality, no payouts, no surpluses credited), $A_{1}$ is a stochastic outcome and depends on the asset allocation and the market returns. Having obtained the values of the assets and the liabilities at time $t=1$, we analyze the distribution of the state of the fund. For this, we first consider the funding ratio $F_{t}$ calculated by dividing the assets by the 
liabilities, i.e.

$$
F_{t}=\frac{A_{t}}{L_{t}}, t=0,1 .
$$

We analyze the mean $\mathbb{E}\left[F_{1}\right]$ as well as selected quantiles $q_{x}$ of $F_{1}$ in order to examine the range of the distribution of the funding ratio at time one in our simulations. We consider the $1 \%, 50 \%$ and $99 \%$ quantiles, denoted by $q_{1 \%}\left(F_{1}\right), q_{50 \%}\left(F_{1}\right)$ and $q_{99 \%}\left(F_{1}\right)$.

Further, we compute the probability of underfunding, i.e. the probability of the funding ratio falling below $100 \%$ at time one. This way, it is measured how exposed to insolvency the fund is. The underfunding probability at time one is defined as

$$
\mathbb{P}\left[F_{1}<100 \%\right] .
$$

The asset returns $\boldsymbol{r}$ are of particular importance, as they influence the allocation $\boldsymbol{\alpha}$ and the distribution of $A_{1}$. For this, it is important to have information about the distribution and the dependency structure of the asset portfolio. For analyzing the returns of the asset portfolio, and subsequently the impact of utility preferences, we examine the first four moments introduced in the following. These first four moments are the expected return $\mu$, the volatility $\sigma$, the skewness $\bar{\gamma}$ and the kurtosis $\bar{\kappa}$ (see, e.g., Bhandari and Das, 2009) of the investment portfolio return.

\subsection{Optimal Portfolio Theory}

In this section, we first present a formulation of a utility function $U(\boldsymbol{\alpha}, \boldsymbol{r})$ based on a limited number of moments of the distribution of $\boldsymbol{r}$. We then consider two particular cases for optimizing the choice of the asset allocation, the minimum variance approach by Markowitz (1952) as well as an alternative one that includes the third and fourth moments of skewness and kurtosis.

\section{Normally Distributed Returns}

In the case of multivariate normally distributed asset returns, the third and fourth moment equal zero. Consequently, the expected utility function equals

$$
\mathbb{E}[U(\boldsymbol{\alpha}, \boldsymbol{r})]=U(\boldsymbol{\alpha}, \boldsymbol{r})+\frac{1}{2} \cdot U^{\prime \prime}(\boldsymbol{\alpha}, \boldsymbol{r}) \cdot \boldsymbol{\alpha}^{\prime} \cdot \Sigma \cdot \boldsymbol{\alpha} .
$$

As the utility function is invariant with respect to positive and monotone transformations (see, e.g., Levy and Markowitz, 1979), we are able to define a function of equivalent utility $V_{1}(\boldsymbol{\alpha}, \boldsymbol{r})$, for which it holds that (see, e.g., Braun et al., 2017)

$$
V_{1}(\boldsymbol{\alpha}, \boldsymbol{r})=\mu-\lambda_{1} \cdot \boldsymbol{\alpha}^{\prime} \cdot \Sigma \cdot \boldsymbol{\alpha}
$$

In this, $\lambda_{1}>0$ implies $U^{\prime \prime}(\boldsymbol{\alpha}, \boldsymbol{r})<0$ and thus serves as a measure for risk aversion. Conversely, $\lambda_{1}<0$ corresponds to a risk taking behavior while $\lambda_{1}=0$ stands for a risk-neutral decision making. Consequently, as $V_{1}(\boldsymbol{\alpha}, \boldsymbol{r})$ is of equivalent utility to $\mathbb{E}[U(\boldsymbol{\alpha}, \boldsymbol{r})]$, the combination of parameters (shares $\alpha$ ) that maximize $V_{1}(\boldsymbol{\alpha}, \boldsymbol{r})$ will also turn out to maximize the expected utility $\mathbb{E}[U(\boldsymbol{\alpha}, \boldsymbol{r})]$.

To find the portfolio that maximizes the utility function, we search for the classic Markowitz (1952) minimum variance portfolio. In this, the aim is to find an optimal set of portfolio weights $\boldsymbol{\alpha}^{*}=\left(\alpha_{1}^{*}, \ldots, \alpha_{n}^{*}\right)^{\prime}$ for the different asset classes that minimizes the variance of the resulting portfolio while achieving a certain target return $\mu^{*}$. We therefore have the constrained optimization problem

$$
\boldsymbol{\alpha}^{*}=\arg \min _{\boldsymbol{\alpha}}\left[\lambda_{1} \cdot \boldsymbol{\alpha}^{\prime} \cdot \Sigma \cdot \boldsymbol{\alpha}\right],
$$


with the objective

$$
\boldsymbol{\alpha}^{\prime} \cdot \boldsymbol{\mu}=\mu^{*},
$$

where

$$
0 \leq \alpha_{i} \leq \alpha_{i}^{\max }, i=1, \ldots, n,
$$

and

$$
\boldsymbol{\alpha}^{\prime} \cdot \mathbb{1}=1
$$

The investment restrictions on $\alpha_{i}$ require that no short sales are made, the share $\alpha_{i}$, that is invested in each asset class, remains below the respective regulatory upper limit $\alpha_{i}^{\max }$ (Equation 10) and the entire assets are invested on the capital market (Equation 11).

Depending on the perspective taken, the objective in Equation (9) is interchangeable with other target conditions considering the funding ratio or the underfunding probability, leading to conditions of the type

$$
\mathbb{E}\left[F_{1}\right]=\bar{F},
$$

where $\bar{F}$ is a given funding ratio target, and

$$
\mathbb{P}\left[F_{1}<100 \%\right]=\epsilon .
$$

where $\epsilon$ is a predetermined one-year probability for underfunding (i.e., $\left.F_{1}<100 \%\right)^{1}$

\section{General Case}

While the optimization problem introduced in Equations $8 \mathrm{ff}$. can be solved relatively easily and yields the minimum variance portfolio, it relies on the assumption that the asset returns follow a multivariate normal distribution. Research shows that in practice, capital market returns do not show Gaussian properties (see, e.g., Jondeau et al., 2007; Cont, 2001). As the historical values exhibit clear signs of asymmetry and heavy tails, it is in many situations consistent for the decision maker to use a valuation technique that higher moments into account. Therefore, the corresponding expected utility function can in general not be simplified to only include the variance of the asset returns. Instead, we aim to find the optimal investment weights $\boldsymbol{\alpha}^{*}$ with respect to the (still approximate) expected utility function. If we again follow the above procedure of applying positive and monotone transformations, we obtain the utility function $V_{2}(\boldsymbol{\alpha}, \boldsymbol{r})$, which is defined as

$$
V_{2}(\boldsymbol{\alpha}, \boldsymbol{r})=\mu-\lambda_{1} \cdot \boldsymbol{\alpha}^{\prime} \cdot \Sigma \cdot \boldsymbol{\alpha}+\lambda_{2} \cdot \boldsymbol{\alpha}^{\prime} \cdot \Gamma \cdot(\boldsymbol{\alpha} \otimes \boldsymbol{\alpha})-\lambda_{3} \cdot \boldsymbol{\alpha}^{\prime} \cdot K \cdot(\boldsymbol{\alpha} \otimes \boldsymbol{\alpha} \otimes \boldsymbol{\alpha}) .
$$

Again, $\lambda_{1}$ serves as a measure for risk aversion, with $\lambda_{1}>0$ being equivalent to $U^{\prime \prime}(\boldsymbol{\alpha}, \boldsymbol{r})<0$. Analogously, the new parameters, $\lambda_{2}$ and $\lambda_{3}$ represent risk preferences (for $\lambda_{2}, \lambda_{3}>0$ ) regarding the third and fourth moments of skewness and kurtosis. As individuals prefer odd moments and try to avoid even ones (see, e.g. Chiu, 2010; Scott and Horvath, 1980), the signs of the terms in $V_{2}(\boldsymbol{\alpha}, \boldsymbol{r})$ alternate. The reasoning behind this is, that the second moment represents the dispersion of the asset returns, which a risk averse investor would aim to keep as low as possible. For the skewness, it holds that a negative skewness corresponds to the mass of the distribution being concentrated on the right with a longer left tail. Correspondingly, a positive third moment has the mass of the distribution shifted towards the left while the right tail is longer. Due to the characteristics of the tails, a risk-averse investor would prefer a positive skewness of the asset returns, as it reduces the risk of extreme losses (low returns). The kurtosis

\footnotetext{
${ }^{1}$ When the investment restrictions on $\alpha_{i}$ from Equation (10) are used, a closed-form solution of the optimization problem can not be derived. We consequently solve the constrained optimization problem using numerical approximations. For further details, see, e.g. Samuelson (1970) and Harvey et al. (2010).
} 
serves as a measure for the tails of the distribution. For this, a large value corresponds to distinctive peaks with little weight on tails, whereas a small value signifies lower peaks and heavy tails. Risk-averse individuals would therefore prefer a smaller kurtosis.

Taking the equivalent utility function $V_{2}(\boldsymbol{\alpha}, \boldsymbol{r})$ into account, Equation (8) of the optimization problem becomes

$$
\boldsymbol{\alpha}^{*}=\arg \min _{\boldsymbol{\alpha}}\left[\lambda_{1} \cdot \boldsymbol{\alpha}^{\prime} \cdot \Sigma \cdot \boldsymbol{\alpha}-\lambda_{2} \cdot \boldsymbol{\alpha}^{\prime} \cdot \Gamma \cdot(\boldsymbol{\alpha} \otimes \boldsymbol{\alpha})+\lambda_{3} \cdot \boldsymbol{\alpha}^{\prime} \cdot K \cdot(\boldsymbol{\alpha} \otimes \boldsymbol{\alpha} \otimes \boldsymbol{\alpha})\right],
$$

with the same target conditions laid out above in Equations (9), (12) or (13), and under the investment restrictions of Equations (10) and (11) on $\boldsymbol{\alpha}$.

In the following, we aim to compare the two optimization problems and their outcomes with each other. This involves fitting distributions to the historical asset return data presented in the following section, as well as determining the covariance, co-skewness and co-kurtosis. Based on those, we aim to compute the optimal solutions of the two optimization problems and consequently analyze their simulation results.

\section{Asset Return Statistics and Distribution}

In order to model the assets that the pension fund can invest in, we use five different asset classes that represent the most common investment types. Those involve the money market, government bonds, real estate, stocks and hedge funds (representing riskier investments). As our research focuses on the Swiss pension fund system, we make use of financial products that are connected to the Swiss market. An overview of the characteristics of the asset classes introduced above is given in Table 1.

\begin{tabular}{lrrrr}
\hline Asset class & Mean return $\mu_{i}$ & Volatility $\sigma_{i}$ & Skewness $\bar{\gamma}_{i}$ & Kurtosis $\bar{\kappa}_{i}$ \\
\hline Money Market (MM) & $1.14 \%$ & $0.34 \%$ & $16.54 \%$ & $19.81 \%$ \\
Government Bonds (GB) & $3.95 \%$ & $3.72 \%$ & $3.74 \%$ & $28.28 \%$ \\
Real Estate (RE) & $5.28 \%$ & $7.07 \%$ & $-12.70 \%$ & $33.34 \%$ \\
Stocks (ST) & $7.27 \%$ & $15.57 \%$ & $-27.13 \%$ & $41.76 \%$ \\
Hedge Funds (HF) & $8.72 \%$ & $7.58 \%$ & $0.45 \%$ & $24.04 \%$ \\
\hline
\end{tabular}

Table 1: Overview of asset classes with their respective annualized mean return $\mu_{i}$, volatility $\sigma_{i}$, skewness $\bar{\gamma}_{i}$ and kurtosis $\bar{\kappa}_{i}$ in the time period $1996-2015 .^{2}$

\subsection{Fitting of Return Distributions}

For simulating the asset returns, the multivariate normal distribution is not able to take the skewness and kurtosis of the returns into account. Furthermore, it is not suited for the modeling of fat tails. We therefore fit the historic data with alternative distributions in order to better describe the empirical distribution. This includes, among others, a better fit of the asymmetry as well as the tails of the returns. The distributions we consider are the Normal, Cauchy, Logistic and Normal-Inverse-Gaussian (NIG) distribution. We use the Akaike information criterion (AIC, see Akaike, 1973) values to evaluate the goodness-of-fit. The results are reported in Table 2.

When comparing the results, we conclude that the NIG distribution provides the best fit on our return data for the money market and the stocks. For the government bonds and the real estate investments

\footnotetext{
${ }^{2}$ As the empirical moments of the third and fourth order are calculated by taking the third and fourth power of the returns, they are particularly sensitive to changes in the data. Different historic values can consequently result in strong changes in the empirical moments. This needs to be taken into account when calculating the skewness and kurtosis.
} 


\begin{tabular}{lrrrrr}
\hline & Money Market & Gvt. Bonds & Real Estate & Stocks & Hedge Funds \\
\cline { 2 - 6 } Normal & -2628.35 & -1485.09 & -1178.72 & -800.79 & -1144.47 \\
Cauchy & -2531.36 & -1410.13 & -1126.66 & -775.29 & -1051.68 \\
Logistic & -2616.45 & -1485.15 & -1186.16 & -816.72 & -1138.91 \\
NIG & -2651.30 & -1482.60 & -1183.97 & -833.06 & -1140.47 \\
\hline
\end{tabular}

Table 2: AIC values from fitting distributions to the historic monthly returns using 20 years of data.

the logistic distribution is suited best. For the hedge funds, the normal distribution achieves the best fit of the data. For the Cauchy distribution, the results are mixed through the considered asset classes. While it is better than the logistic and the NIG distribution for the hedge funds, it is not better than the normal distribution for any of the asset classes.

\section{Numerical Application}

In the following, we present the results of our simulations for a fund operating in Switzerland. Therein, we initially look at the reference case of multivariate normally distributed assets in a minimum variance portfolio under the utility function $V_{1}(\boldsymbol{\alpha}, \boldsymbol{r})$. Following that, we look at the case of the portfolio weights being chosen in line with the extended utility function $V_{2}(\boldsymbol{\alpha}, \boldsymbol{r})$ and the returns following the fitted distributions. We examine how the asset allocation changes and analyze the outcome of the simulations. The risk factors $\lambda_{1}, \lambda_{2}$ and $\lambda_{3}$ are set to one in these cases. The numerical application is performed by simulating the efficient portfolios for target values between $\min \left(\mu_{i}\right)=1.14 \%$ and the maximum attainable return of $6.73 \%$ using a step size of $0.1 \%$ and $N=10^{7}$ (10 million) realizations for every simulation.

With respect to the investment limits, $\alpha_{i}^{\max }$, there are no specific limitations for the shares invested in the money market and the government bonds. For the real estate, the maximum share, that can be invested, amounts to $30 \%$. Conversely, the proportion of the assets, that is made up by the stocks, can be up to $50 \%$. The strictest limit is imposed on the hedge funds. For them, the maximum share amounts to $15 \%$. For our simulations, we therefore use the vector $\boldsymbol{\alpha}^{\max }=(100 \%, 100 \%, 30 \%, 50 \%, 15 \%)^{\prime}$ for the investment limits (see BVV2, 2017, Art. 55). Furthermore, we assume that the fund starts in a "healthy" situation with assets of $A_{0}=110$ and liabilities of $L_{0}=100$ at time $t=0$. The funding ratio $F_{0}=A_{0} / L_{0}$ consequently equals $110 \%$, which corresponds to the average value for private pension funds in Switzerland over the past years (see Swisscanto, 2018). While the return on the assets $r_{\mathrm{A}}$ is random following the distributions laid out in Section 3, the interest rate $r_{\mathrm{L}}$ for the liabilities is set to the legal minimum. For 2016, it equals 1.25\% (see BVV2, 2017, Art. 12).

\subsection{Classic Markowitz Optimization with Normally Distributed Returns}

Serving as a reference case, we first simulate the pension fund using the asset allocation derived from minimum variance theory. Therein, the asset classes are multivariate normally distributed and the risk aversion coefficient $\lambda_{1}=1$. Figure 1 displays the asset allocations in the efficient portfolios for the restricted case.

For every combination of target returns and corresponding minimum variances, it depicts the optimal shares $\boldsymbol{\alpha}^{*}$ of the five asset classes. It can be seen that for low target returns the efficient portfolio mainly consists of money market investments. As the drift $\mu$ increases, this share decreases while the shares of the remaining assets increase, with the government bonds having the second-highest percentage. As $\mu$ grows further, the share of the hedge funds and the government bonds evolve similarly at first. The 


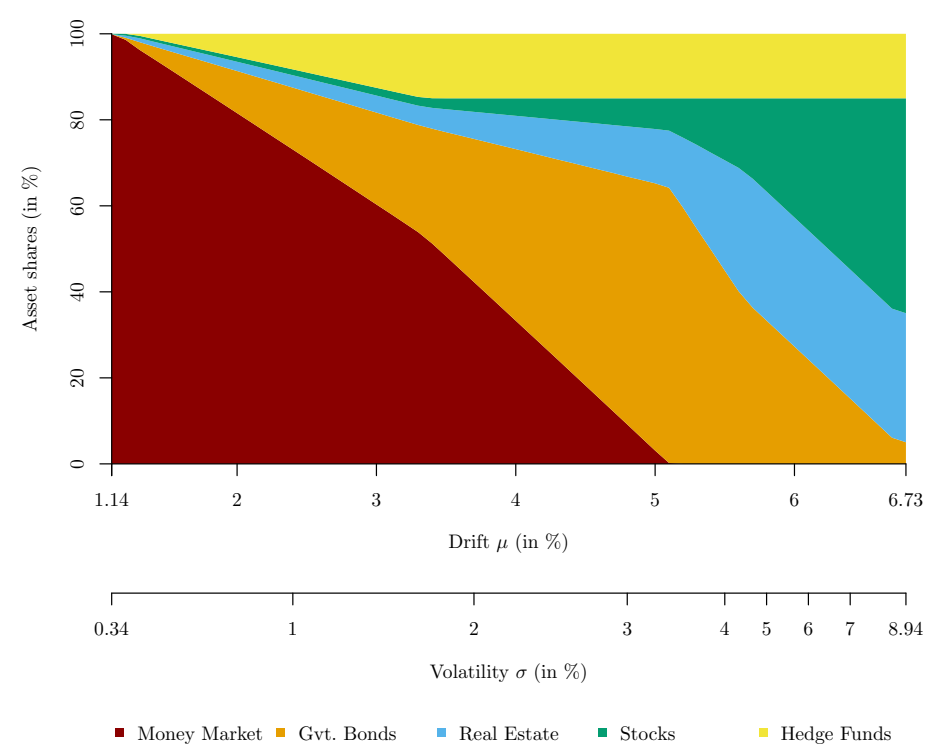

Figure 1: Optimal asset allocations for target return $\mu$ for $V_{1}(\boldsymbol{\alpha}, \boldsymbol{r})$ and normally distributed returns.

stocks are hardly represented in the portfolio. The changes in the asset shares are linear up to a drift of almost $3.5 \%$, where the hedge funds reach their maximum of $\alpha_{5}^{\max }=15 \%$. Past this point, the share of the money market begins to decrease steeply and the portfolio weights shift more towards the government bonds, the real estate and the stocks. For the stocks, it can be seen that an increase of its share is taking place as a result of the hedge funds hitting their investment limit. The same holds true for the real estate. For the government bonds a decrease takes place for high returns. For the portfolio with the highest return, which is located on the right end of the graph, the hedge funds, the stocks and the real estate achieve their investment limits of $15 \%, 50 \%$ and $30 \%$. The remaining proportion of $5 \%$ is attributed to the government bonds. The portfolio weights thus are $\boldsymbol{\alpha}=(0 \%, 5 \%, 30 \%, 50 \%, 15 \%)^{\prime}$, which means that the highest achievable portfolio return is $6.73 \%$ along with a volatility of $8.94 \%$. Overall, changes of the shares take place with kinks in the graphs when assets hit their investment limits or disappear from the portfolio. The numerical results for selected target values are given in Table 3 . In the three parts of the table, we fix target values for the mean return (part I), the expected funding ratio $\mathbb{E}\left[F_{1}\right]$ at time one (part II) and the one-year underfunding probability $\mathbb{P}\left[F_{1}<100 \%\right]$ (part III). These objectives correspond to the conditions described in Equations (9), (12) and (13).

\subsection{Asset Allocation with Best-Fit Distributed Returns}

In the previous section, we reported the investment portfolios when using the utility function $V_{1}(\boldsymbol{\alpha}, \boldsymbol{r})$ and normally distributed returns. In the following, we depart this approach and look at the results when using the utility function $V_{2}(\boldsymbol{\alpha}, \boldsymbol{r})$ integrating the first four moments of the returns in each asset class and when considering the return distributions that fit the historic returns best for simulating the values at time one (cf. Section 3.1). This way, we aim to find out how using moments of higher order in the utility function and more suitable return distributions alter the previously obtained results. In order to ensure comparability, the parameter values at time zero and the risk factors remain unchanged. Figure 2 depicts the asset shares in the optimal portfolios for $V_{2}(\boldsymbol{\alpha}, \boldsymbol{r})$ with returns that are in agreement with the best-fit distributions. It can directly be compared with Figure 1.

As before, the portfolio with the smallest drift consists only of the money market. When increasing 


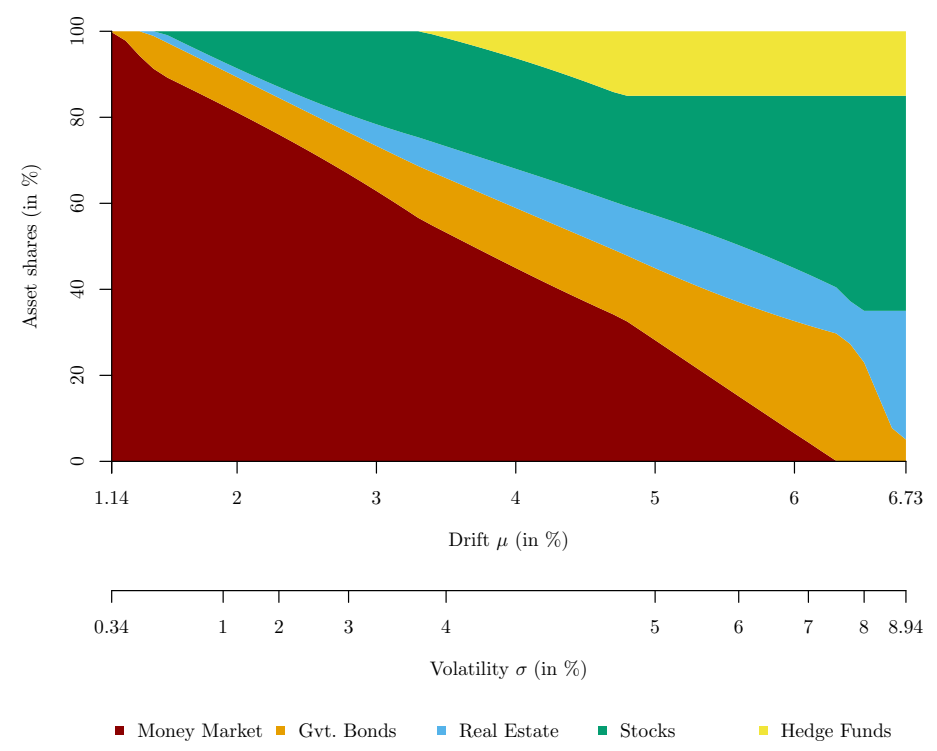

Figure 2: Optimal asset allocations for target return $\mu$ for $V_{2}(\boldsymbol{\alpha}, \boldsymbol{r})$ and best-fit distributed returns.

the portfolio return from this point on, we can see that at first only the government bonds are added to the portfolio. After this, an investment in stocks takes place. Up to a drift of $4 \%$, their share grows up to about $25 \%$. Meanwhile, the real estate and the hedge funds remain at a single-digit value. From $\mu=3.5 \%$, the share of the hedge funds begins to increase quickly. Once they reach their maximum value, the stocks increase their share again. This continues until they reach their maximum percentage of $\alpha_{4}^{\max }=50 \%$. Subsequently, the real estate replaces the government bonds up to their respective investment limit of $\alpha_{3}^{\max }=30 \%$. The portfolio with the highest drift and return consequently is the same as in the minimum variance case. The course of the portfolio volatility $\sigma$ is given on the second horizontal axis in Figure 2. There, it can be seen that the increase of $\sigma$ is not linear. Up to a portfolio return of about $3.5 \%$ the volatility increases uniformly. Past that point, more weight is put on the hedge funds which have a lower dispersion. The growth of the portfolio volatility consequently slows down. Once the hedge funds reach the maximum share $\alpha_{5}^{\max }$, the percentages of the remaining assets

\begin{tabular}{l|rrr|rrr|rrr}
\hline Condition on & \multicolumn{3}{|c|}{ I: Mean return } & II: Expected funding ratio & III: Underfunding probability \\
\hline$\mu$ & $\mathbf{2 . 0 0}$ & $\mathbf{3 . 0 0}$ & $\mathbf{4 . 0 0}$ & 1.25 & 3.05 & 4.82 & 5.73 & 6.04 & 6.21 \\
$\sigma$ & 0.74 & 1.48 & 2.29 & 0.34 & 1.52 & 3.06 & 4.75 & 5.89 & 6.58 \\
$\bar{\gamma}\left[\times 10^{-2}\right]$ & 0.11 & 0.13 & 0.12 & 0.01 & 0.13 & 0.11 & 0.25 & 0.32 & 0.35 \\
$\bar{\kappa}\left[\times 10^{-2}\right]$ & 0.03 & 0.03 & 0.03 & 0.00 & 0.04 & 0.02 & 0.13 & 0.21 & 0.24 \\
\hline$\alpha_{1}$ & 81.56 & 60.18 & 33.28 & 97.21 & 59.17 & 8.48 & 0.00 & 0.00 & 0.00 \\
$\alpha_{2}$ & 9.78 & 21.57 & 39.90 & 1.42 & 22.02 & 58.18 & 35.48 & 26.18 & 21.06 \\
$\alpha_{3}$ & 2.16 & 3.94 & 7.75 & 0.76 & 4.04 & 11.75 & 30.00 & 30.00 & 30.00 \\
$\alpha_{4}$ & 1.07 & 1.85 & 4.07 & 0.56 & 1.85 & 6.59 & 19.52 & 28.82 & 33.94 \\
$\alpha_{5}$ & 5.44 & 12.46 & 15.00 & 0.06 & 12.92 & 15.00 & 15.00 & 15.00 & 15.00 \\
\hline $\mathbb{E}\left[F_{1}\right]$ & 110.83 & 111.94 & 113.07 & $\mathbf{1 1 0 . 0 0}$ & $\mathbf{1 1 2 . 0 0}$ & $\mathbf{1 1 4 . 0 0}$ & 115.04 & 115.39 & 115.59 \\
$q_{1 \%}\left(F_{1}\right)$ & 108.94 & 108.19 & 107.28 & 109.14 & 108.15 & 106.30 & 103.46 & 101.34 & 100.00 \\
$q_{50 \%}\left(F_{1}\right)$ & 110.86 & 112.00 & 113.18 & 110.01 & 112.06 & 114.16 & 115.26 & 115.60 & 115.78 \\
$q_{99 \%}\left(F_{1}\right)$ & 112.95 & 116.22 & 119.64 & 110.89 & 116.39 & 122.73 & 129.48 & 133.78 & 136.35 \\
\hline $\mathbb{P}\left[F_{1}<100 \%\right]$ & 0.00 & 0.00 & 0.00 & 0.00 & 0.00 & 0.00 & $\mathbf{0 . 1 0}$ & $\mathbf{0 . 5 0}$ & $\mathbf{1 . 0 0}$ \\
\hline
\end{tabular}

Table 3: Simulation results using minimum variance and normal returns. Values in bold face correspond to the target values. All reported values are given in $\%$. 
in the optimal portfolio increase again and the portfolio volatility returns to increasing more strongly. Simulation results for different cases are presented in Table 4. As before, we have three parts where we

\begin{tabular}{l|rrr|rrr|rrr}
\hline Condition on & \multicolumn{3}{|c|}{ I: Mean return } & \multicolumn{2}{|c|}{ II: Expected funding ratio } & III: Underfunding probability \\
\hline$\mu$ & $\mathbf{2 . 0 0}$ & $\mathbf{3 . 0 0}$ & $\mathbf{4 . 0 0}$ & 1.24 & 3.03 & 4.76 & 3.04 & 4.88 & 5.29 \\
$\sigma$ & 1.36 & 3.40 & 4.32 & 0.37 & 3.41 & 4.62 & 3.47 & 4.85 & 5.55 \\
$\bar{\gamma}\left[\times 10^{-2}\right]$ & 0.09 & 0.10 & 0.10 & 0.24 & 0.10 & 0.10 & 0.10 & 0.10 & 0.10 \\
$\bar{\kappa}\left[\times 10^{-2}\right]$ & 0.09 & 0.10 & 0.09 & 0.15 & 0.10 & 0.09 & 0.10 & 0.09 & 0.09 \\
\hline$\alpha_{1}$ & 81.12 & 62.86 & 44.96 & 96.32 & 62.12 & 32.99 & 62.06 & 30.78 & 21.87 \\
$\alpha_{2}$ & 8.22 & 10.48 & 13.97 & 3.68 & 10.62 & 15.03 & 10.66 & 15.86 & 19.06 \\
$\alpha_{3}$ & 2.04 & 5.03 & 9.10 & 0.00 & 5.68 & 12.25 & 5.23 & 11.81 & 13.06 \\
$\alpha_{4}$ & 8.62 & 21.63 & 25.72 & 0.00 & 21.58 & 24.92 & 22.05 & 26.54 & 31.01 \\
$\alpha_{5}$ & 0.00 & 0.00 & 6.26 & 0.00 & 0.00 & 14.81 & 0.00 & 15.00 & 15.00 \\
\hline $\mathbb{E}\left[F_{1}\right]$ & 110.83 & 111.94 & 113.07 & $\mathbf{1 1 0 . 0 0}$ & $\mathbf{1 1 2 . 0 0}$ & $\mathbf{1 1 4 . 0 0}$ & 111.99 & 114.07 & 114.54 \\
$q_{1 \%}\left(F_{1}\right)$ & 107.26 & 103.05 & 101.76 & 109.09 & 103.06 & 101.83 & 102.91 & 101.36 & 100.00 \\
$q_{50 \%}\left(F_{1}\right)$ & 110.96 & 112.25 & 113.48 & 109.99 & 112.29 & 114.39 & 112.30 & 114.54 & 115.07 \\
$q_{99 \%}\left(F_{1}\right)$ & 114.93 & 122.15 & 126.08 & 111.06 & 122.21 & 127.91 & 122.41 & 128.73 & 131.30 \\
\hline $\mathbb{P}\left[F_{1}<100 \%\right]$ & 0.00 & 0.08 & 0.35 & 0.00 & 0.08 & 0.37 & $\mathbf{0 . 1 0}$ & $\mathbf{0 . 5 0}$ & $\mathbf{1 . 0 0}$ \\
\hline
\end{tabular}

Table 4: Simulation results using extended utility and alternative returns. All values are given in \%.

set target values for the mean return, the expected funding ratio and the underfunding probability. In order to ensure the comparability of the results, we utilize the same target values as in Table 3.

\section{Conclusion}

In this research, we look at the impact that higher moments have on the optimal asset allocation of a pension fund. The research question was: To what extend do (1) higher moments in the optimization and (2) best-fit return distributions for the simulations lead to a change in the investment portfolio weights. To this end, we compare how the Markowitz efficient portfolio asset weights change when using an extended utility function that also considers the third and fourth moments of the historic asset returns. Using the so obtained portfolio weights, we simulate the assets of the fund in a one-period model. Therein, normally distributed returns are compared to alternative ones that fit the data in a better way. Furthermore, it is examined how a more complex risk preference with respect to several return moments impacts the optimization results. In order to deepen the analysis, a sensitivity analysis in which the different risk preference factors are varied is presented in the full paper by Müller and Wagner (2018). In our work we consider three different key indicators that are relevant for pension fund management in practice: the target return, the expected one-period funding ratio and the one-period underfunding probability.

Expectedly, our results indicate that the use of an extended utility function does indeed lead to a shift in the optimal portfolio weights. Consequently, main portfolio characteristics change. Among others, a strong increase of the volatility takes place in our example. Following this, the outer quantiles of the funding ratio spread further and the underfunding probability increases. We are therefore able to say that using the minimum variance portfolio can cause misleading security. Working with an extended utility function that departs from the minimum variance framework and incorporates higher moments of returns consequently may direct companies to assess their risk taking more adequately.

Along with the utility function, pension funds should also consider looking at adequate distributions 
for their asset classes. As previous research shows, the normal distribution is not able to fully reflect the characteristics of asset returns. Comparing it to three other distributions, we are able to find a distribution that fits the historic return data better for almost every type of assets. Using these, we simulate returns that are not symmetric and put more weight on the tails, properties that are characteristic for capital market returns. Consequently, the characteristics of the optimal portfolios such as the skewness and kurtosis change in our simulations. In particular, the return distribution alters to having a longer right tail and being less peaked. We are therefore of the opinion that pension funds, and financial institutions in general, need to consider the most suitable distributions when simulating their asset values.

While the "best" asset allocation is still to be interpreted in the light of the used hypotheses and objectives, the investment shares - differing little or a lot - from the different methods give information about the stability and robustness of the calculated allocations. While being focused on the pension fund system, our results hold true for financial institutions in general. Practical implementation is often impeded by the high number of parameters that must be estimated and set as input. Further results, sensitivity analyses and comments can be found in Müller and Wagner (2018).

\section{References}

Akaike, H., 1973, Information Theory and an Extension of the Maximum Likelihood Principle, International Symposium on Information Theory, pages 267-281.

Bhandari, R. and S. R. Das, 2009, Options on Portfolios with Higher-Order Moments, Finance Research Letters, 6(3):122-129.

Braun, A., H. Schmeiser, and F. Schreiber, 2017, Portfolio Optimization Under Solvency II: Implicit Constraints Imposed by the Market Risk Standard Formula, Journal of Risk and Insurance, 84(1):177207.

BVV2, 2017, Verordnung über die berufliche Alters-, Hinterlassenen- und Invalidenvorsorge, (1 April 2018), from https://www.admin.ch/opc/de/classified-compilation/19840067/index.html.

Chiu, W. H., 2010, Skewness Preference, Risk Taking and Expected Utility Maximisation, The Geneva Risk and Insurance Review, 35(2):108-129.

Cont, R., 2001, Empirical Properties of Asset Returns: Stylized Facts and Statistical Issues, Quantitative Finance, 1(2):223-236.

Harvey, C. R., J. C. Liechty, M. W. Liechty, and P. Müller, 2010, Portfolio Selection with Higher Moments, Quantitative Finance, 10(5):469-485.

Jondeau, E., S.-H. Poon, and M. Rockinger, 2007, Financial Modeling Under Non-Gaussian Distributions. Springer Finance. Springer London.

Levy, H. and H. M. Markowitz, 1979, Approximating Expected Utility by a Function of Mean and Variance, The American Economic Review, 69(3):308-317.

Markowitz, H., 1952, Portfolio Selection, The Journal of Finance, 7(1):77-91.

Müller, P. and J. Wagner, 2018, Optimal Asset Allocation in Pension Funds Under Consideration of Higher Moments, Working paper, University of Lausanne. 
Samuelson, P. A., 1970, The Fundamental Approximation Theorem of Portfolio Analysis in Terms of Means, Variances and Higher Moments, The Review of Economic Studies, 37(4):537-542.

Scott, R. C. and P. A. Horvath, 1980, On the Direction of Performance for Moments of Higher Order Than the Variance, Journal of Finance, 35(4):915-919.

Swisscanto, 2018, Pensionskassen-Monitor 2017, from http://www. swisscanto.ch/pk-monitor. 